\title{
Editorial
}

\section{El ciberriesgo, \\ tensión para los \\ saberes previos}

DOI: 10.29236/sistemas.n151a1

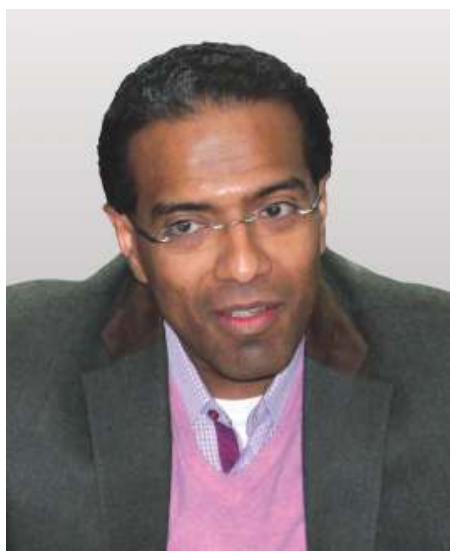

Un actor emergente que surge más allá del ambiente de negocios e involucra al ser humano en su dimensión completa.

\section{Jeimy J. Cano M.}

La acelerada transformación de las lacional, en la que ya no son los elementos del sistema los que tienen empresas y de la sociedad, acomel protagonismo, sino sus interacpañada del incremento de la densidad digital de los objetos físicos, ciones y conexiones conocidas (y establece un nuevo paradigma de comprensión social y económico, que debilita la visión mecanicista del mundo y privilegia una más reemergentes).

En este contexto, la comprensión actual de los riesgos, asociada con 
aquellos conocidos y controlables, le da paso a una nueva lectura que revela, en un escenario hiperconectado, situaciones que pueden ser latentes o desconocidas para las organizaciones y cuyo tratamiento no responde a estándares vigentes a la fecha.

En este sentido, el concepto de ciberriesgo como una realidad sistémica (relacional, dinámica y evolutiva) genera una nueva frontera de reflexiones para los profesionales de seguridad y control, así como, para los ejecutivos de una organización, que los invita a realizar una categorización de los riesgos distinta a la tradicional. Para lograrlo, ahora ellos deben partir de "en relación con" y no "de acuerdo con", retando los estándares vigentes como ISO 31000 e ISO 27005 -para citar algunos-, diseñados para entender entornos conocidos.

Es por esto que la revista "Sistemas", de la Asociación Colombiana de Ingenieros de Sistemas -ACIS-, ha decidido revisar en contexto la comprensión, la gestión y el aseguramiento de los ciberriesgos en las organizaciones actuales. Con ese objetivo, fueron convocados profesionales de distintas disciplinas, quienes desde su área de experiencia proponen reflexiones para seguirle la pista al ejercicio sistémico, en el marco de la convergencia tecnológica, las inestabilidades y las posibilidades de un mundo cada vez más digital y tecnológicamente modificado.
El ingeniero Juan Mario Posada Daza, columnista invitado, establece desde su práctica de consultoría un marco base para reflexionar sobre el ciberriesgo, como el reto de la interdependencia de las empresas en el ciberespacio, que hace de la cooperación un fundamento clave para frenar las situaciones de ataque y responder a los hechos que podrían desestabilizar empresas y naciones. En esa dirección presenta una revisión de documentos de la práctica internacional, en los que fundamenta sus posturas sistémicas y el reconocimiento del ciberriesgo como un elemento estratégico y de negocio en las organizaciones modernas.

Por su parte, el ingeniero Andrés Almanza Junco presenta el análisis de los resultados de la décimo novena encuesta nacional de seguridad de la información, realizada cada año por ACIS, estudio que revela las tendencias más representativas de las empresas colombianas en los temas de protección de la información y la evolución del líder digital de seguridad, así como sus respectivos contrastes con la realidad internacional. Contempla una serie de reflexiones encaminadas al análisis alrededor de la evolución de la seguridad de la información, además del estado actual de la práctica de seguridad y control en el país.

El tradicional foro de la revista contó con la participación de destacados profesionales involucrados con 
los retos propios de los ciberriesgos, quienes intercambiaron conceptos y cuestionaron las fronteras actuales de su tratamiento, además de analizar la comprensión de la convergencia tecnológica y sus implicaciones en los negocios. Ellos advierten sobre la necesidad de reinventar la práctica de la gestión de riesgos y amenazas dentro de las organizaciones frente a un escenario cada vez más disruptivo, inestable e hiperconectado, ambiente que demanda una mayor anticipación que prevención.

Así mismo, nuestros lectores dispondrán de dos artículos para reflexionar sobre la conceptualización y gestión del ciberriesgo. Un primer documento de autoría de este servidor se ocupa de los fundamentos del ciberriesgo, con el propósito de ilustrar tres características clave que lo definen como sistémico, emergente y disruptivo. Dicho ejercicio apunta hacia una mirada sistémica y las implicaciones que su materialización puede tener en un escenario hiperconectado. El segundo artículo, escrito por el ingeniero Joshsua González Díaz, aborda el ciberriesgo desde una perspectiva técnica con las implicaciones económicas y sociales. El autor detalla algunos análisis de este riesgo en el sector financiero y plantea varios elementos de cara al concepto de ciberresiliencia.

De manera que, este número de la revista "Sistemas" ofrece un panorama renovado de análisis y consideraciones sobre un nuevo tipo de riesgo, que tensiona los saberes y prácticas existentes. Su contenido invita a todos los profesionales en las diferentes áreas, a mirar de forma convergente las nuevas realidades que revela un mundo digital y tecnológicamente modificado, sin perjuicio de los nuevos desafíos técnicos y administrativos, donde los terceros de confianza, hacen realidad capacidades inexistentes en las organizaciones actuales.

Jeimy J. Cano M., Ph.D, CFE, CICA. Profesor Distinguido de la Facultad de Derecho. Universidad de los Andes. Ingeniero y Magíster en Ingeniería de Sistemas y Computación por la Universidad de los Andes. Especialista en Derecho Disciplinario por la Universidad Externado de Colombia. Ph.D en Business Administration por Newport University, CA. USA. y Ph.D en Educación por la Universidad Santo Tomás. Profesional certificado como Certified Fraud Examiner (CFE), por la Association of Certified Fraud Examiners y Certified Internal Control Auditor (CICA) por The Institute of Internal Controls. Es director de la Revista Sistemas de la Asociación Colombiana de Ingenieros de Sistemas-ACIS-. 